\title{
Simultaneous Kissing Stenting: A Valuable Technique for Reconstructing the Stenotic Initial Segment of the Right Subclavian Artery
}

\author{
Ping Zhang Daiqi Chen Daishi Tian Qiang Zhang Minghuan Wang \\ Qian Li Xiang Luo
}

Department of Neurology, Tongji Hospital, Tongji Medical College, Huazhong University of Science and Technology, Wuhan, PR China

\section{Key Words}

Interventional therapy · Multiple angiostenosis · Simultaneous kissing stenting · Subclavian artery

\begin{abstract}
Atherosclerotic stenosis or occlusion often involves the subclavian artery. For lesions that are close to the orifice of the right subclavian artery, stenting of the right subclavian artery itself blocks the pathway from the innominate artery to the right carotid artery and causes problems in patients with multiple angiostenosis, especially involving the right carotid system. In this study, we report 2 cases using simultaneous kissing stenting (SKS) of the right subclavian artery and the right carotid artery to relieve right subclavian stenosis and maintain right carotid system patency. Standard stenting methods were used to perform SKS. Two self-expanding stents were implanted simultaneously into the initial segment of the right subclavian artery and the right carotid artery, forming a " $Y$ " shape, with the overlap of the proximal segments in the innominate artery $\geq 5 \mathrm{~mm}$. After SKS, the stenosed right subclavian artery was dilated, and the patency of the right carotid system was maintained. The symptoms of patients were relieved and the stents were intact at several months of follow-up. In conclusion, SKS of the right subclavian artery and the right carotid artery might be a safe and effective procedure when the stenotic or occlusive lesion in the initial segment of the right subclavian artery is close to the orifice, and lesions (or potential ones) exist in the right carotid system.
\end{abstract}

(c) 2017 S. Karger AG, Basel 


\section{Introduction}

The majority of the supra-aortic atherosclerotic occlusive lesions involve the subclavian artery [1]. In recent decades, percutaneous transluminal angioplasty, either with or without stent placement, has been considered as the first-line therapy for subclavian obstruction [2]. In many cases, when the stenosis was close to the orifice of the right subclavian artery, only stenting of the right subclavian would be performed. To cover the whole lesion, the proximal end of the stent would be inserted into the innominate artery. As a result, the pathway from the innominate artery to the right carotid artery would be blocked. This presents a problem to patients who have multiple angiostenosis, especially lesions of the right carotid system that might need future stenting. In order to resolve this problem, we performed simultaneous kissing stenting (SKS) of the right subclavian artery and the right carotid artery, thereby successfully maintaining the patency of the right carotid system.

\section{Materials and Methods}

\section{Diagnosis}

The patient's general information, medical history, cardinal symptoms, physical, and imaging examination were collected for the appropriate clinical diagnosis. Digital subtraction angiography, including the 3D run, was used for determining the patient's cerebrovascular condition. Digital subtraction angiography results should fully explain the patient's symptoms and signs. Patients with a stenotic or occlusive lesion in the initial segment of the right subclavian artery, which was close to the orifice, and lesions (or potential ones) in the right carotid system, were included in this study.

Aspirin (100 mg/day), clopidogrel hydrogen sulfate (75 mg/day), and atorvastatin calcium $(20 \mathrm{mg} /$ day) were administered for 7 days before performing angioplasty. The procedure was carried out with local anesthesia in our hospital's neuroangiography suite. Two 8F sheaths were placed percutaneously in the right and left femoral artery separately using the modified Seldinger technique. Using a coaxial catheter system, a 0.035 -inch stiff guidewire, $300 \mathrm{~cm}$ long, was directed through the sheath in the right femoral artery to the distal segment of the right subclavian artery. Then, a $5 \mathrm{~F}$ multipurpose catheter and an $8 \mathrm{~F}$ guiding catheter were advanced along the stiff guidewire to the initial segment of the right subclavian artery. After that, the stiff guidewire was pulled out. A PT 0.014-inch guidewire, $300 \mathrm{~cm}$ long, was inserted into the distal segment of the right subclavian artery through the multipurpose catheter. Then, the multipurpose catheter was pulled out. Under road-map guidance, a balloon catheter (size dependent on the lesion) was navigated through the stenosis of the subclavian artery along the PT 0.014-inch guidewire without distal embolic protection. Thereafter, the balloon angioplasty was performed (pressure and times dependent on the lesion). After that, the balloon catheter was exchanged for a self-expanding stent (size dependent on the lesion), which was not released immediately. In the same way, a PT 0.014-inch guidewire, $185 \mathrm{~cm}$ long, was inserted into the C3 segment of the right internal carotid artery through the sheath in the left femoral artery. Another self-expanding stent was delivered to the initial segment of the right common carotid artery along the guidewire. After checking the locations, both stents were deployed in succession. The 2 stents formed a "Y" shape, with the overlap of the proximal segments in the innominate artery $\geq 5 \mathrm{~mm}$.

\section{Patient 1}

A 57-year-old male with a history of systemic hypertension suffered from recurrent and aggravated vertigo, limbs weakness, and visual field impairment. MRI revealed infarctions of the occipital lobe, thalamus, brainstem, and internal temporal lobe of both sides (Fig. 1). Angiography showed multiple angiostenosis, including stenosis of the left renal artery (about 70\%), the left vertebral artery (about 70\%), the initial segment of the right subclavian artery (about 90\%), as well as mild stenosis of the right common carotid artery (Fig. 2a, b). Subclavian and carotid artery stents were placed simultaneously using SKS technique. Two $9 \times 40 \mathrm{~mm}$ Carotid Wallstents ${ }^{\mathrm{TM}}$ were implanted into the initial segment of the right subclavian artery and the right common carotid artery (Fig. 2c, d). Postoperative angiography revealed excellent dilatation of the stenotic right subclavian artery. However, the right common carotid stent became foreshortened during the 
Zhang et al:: Simultaneous Kissing Stenting: A Valuable Technique for Reconstructing the Stenotic Initial Segment of the Right Subclavian Artery
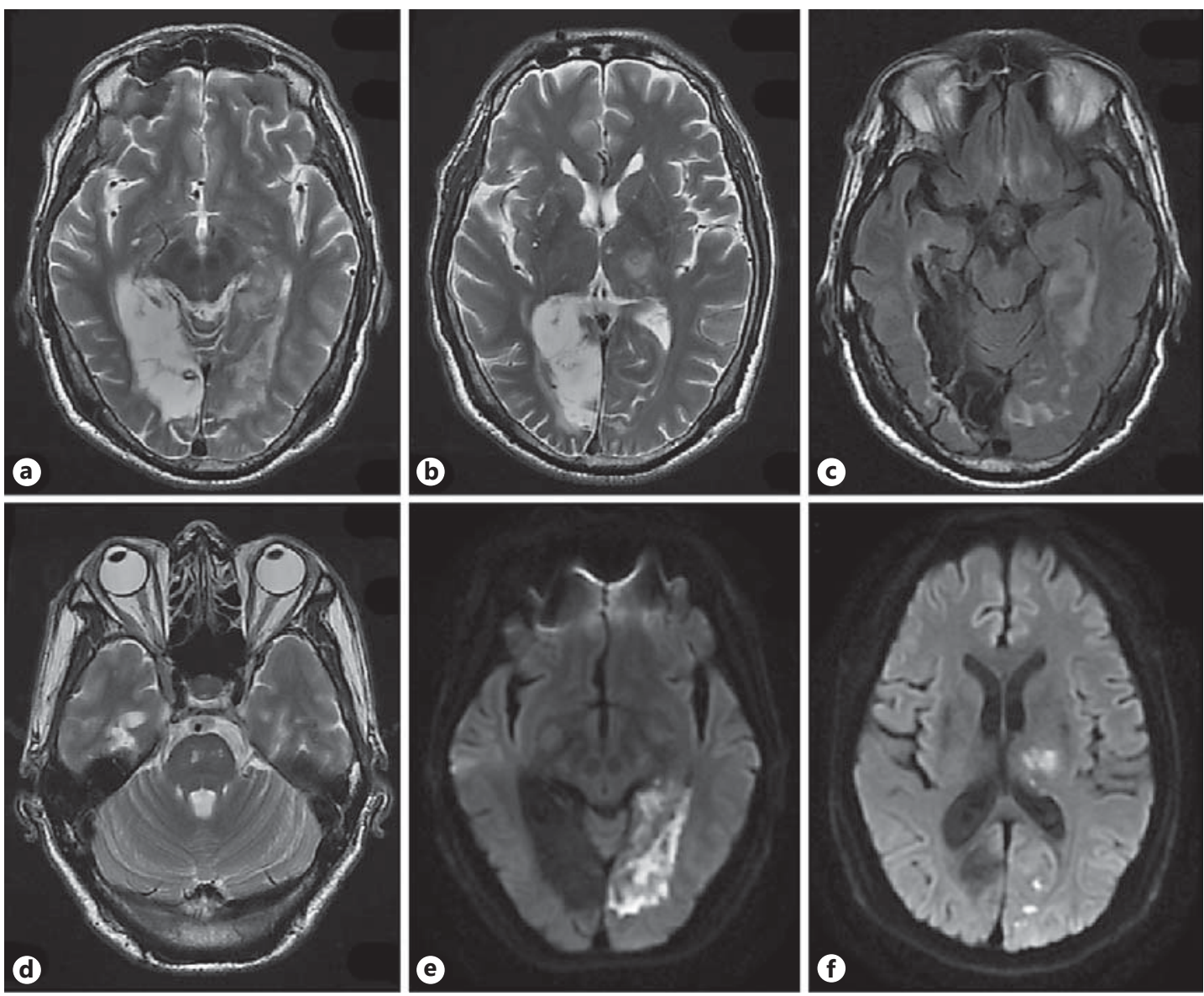

Fig. 1. MRI of Patient 1, revealing infarctions of the occipital lobe, thalamus, brainstem, and internal temporal lobe of both sides (a, b, d T2; c T2 Flair). e, f Diffusion-weighted image showing fresh infarctions of the left occipital lobe and left thalamus.

self-expanding process, which prevented the alignment of the proximal ends of the 2 stents in the innominate artery (Fig. 2e, f). The patient's symptom of vertigo was relieved. And no new infarction was found at 30 months of follow-up.

\section{Patient 2}

A 71-year-old male with a history of hyperlipidemia and lacunar infarction suffered recurrent and aggravated vertigo and weakness of the left limbs. MRI revealed multiple lacunar infarctions (Fig. 3). Angiography showed multiple angiostenosis, including severe stenosis (about 90\%) of the initial segment of the right subclavian artery and severe stenosis (about $85 \%$ ) of the initial segment of the right internal carotid artery, accompanied by ulceration and thrombosis (Fig. 4a, b). Firstly, a $9 \times 30 \mathrm{~mm}$ Carotid Wallstent was used to reconstruct the lumen of the right internal carotid artery under the protection of a FilterWire. Then, a $9 \times 40 \mathrm{~mm}$ and another $10 \times 40 \mathrm{~mm}$ Protégé RX stents were implanted into the initial segment of the right subclavian artery and the right common carotid artery using the SKS technique (Fig. 4c, d). Postoperative angiography revealed excellent dilatation of the stenosis. The proximal ends of the 2 stents were in the innominate artery and aligned (Fig. 4e, f). The symptoms were relieved, and the patient was intact neurologically at 5 months of follow-up. CT angiography performed at 5 months of follow-up showed excellent dilatation of the stenotic segments of the right subclavian artery and the right internal carotid artery, as well as good positions of all stents (Fig. 5). 
Zhang et al.: Simultaneous Kissing Stenting: A Valuable Technique for Reconstructing the Stenotic Initial Segment of the Right Subclavian Artery
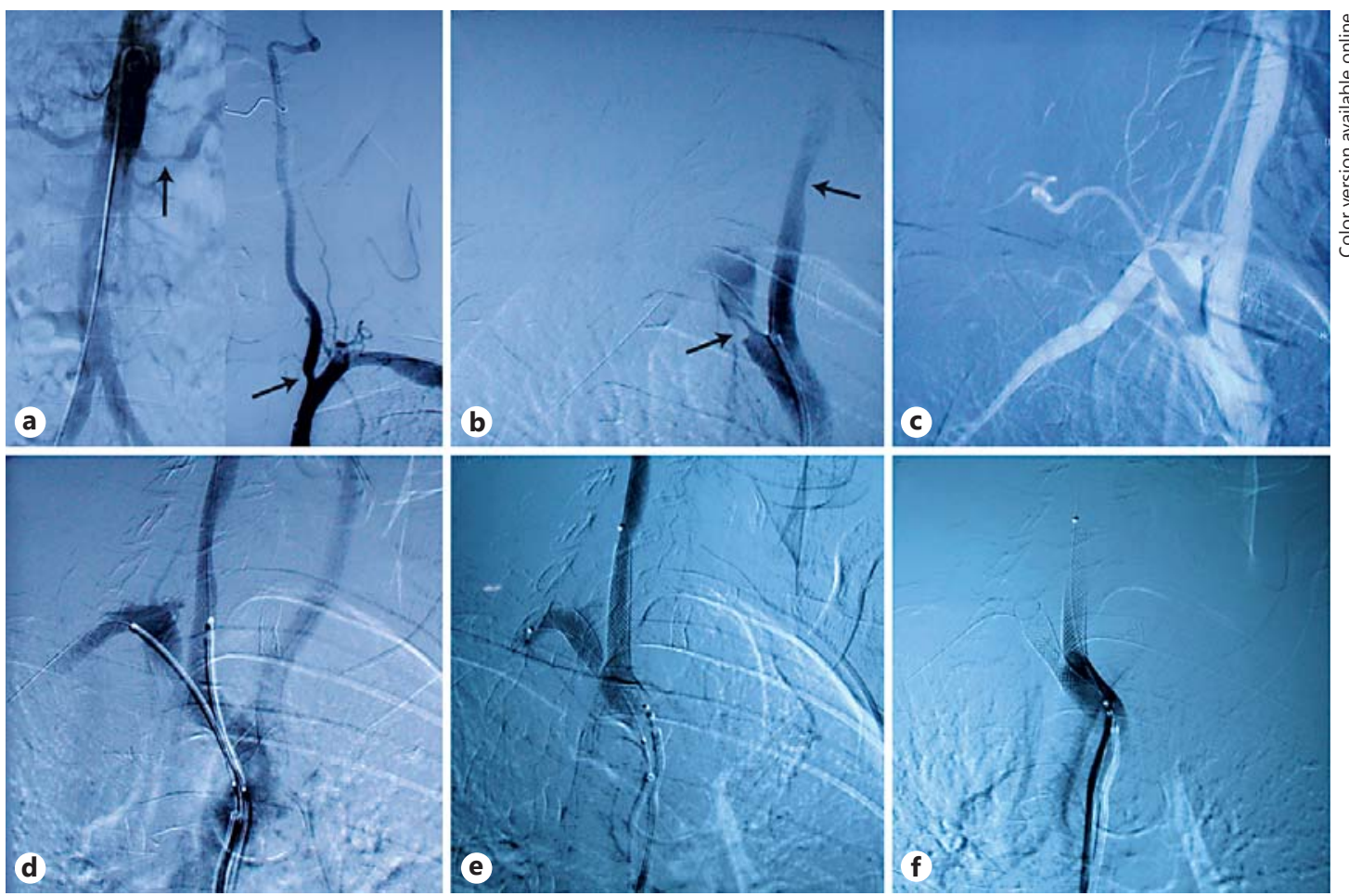

Fig. 2. Patient 1. Angiography showing stenosis (about 70\%) of the left renal artery (left arrow) and stenosis (about 70\%) of the initial segment of the left vertebral artery (right arrow; a); severe stenosis (about 90\%) of the initial segment of the right subclavian artery (left arrow) and mild stenosis of the right common carotid artery (right arrow; b); balloon angioplasty of the stenotic segment of the right subclavian artery (c); two $9 \times 40 \mathrm{~mm}$ Carotid Wallstents ${ }^{\mathrm{TM}}$ inserted into the initial segment of the right subclavian artery and the right common carotid artery (d); excellent dilatation of the stenotic segment of the right subclavian artery after SKS (e), and nonalignment of the proximal ends of the 2 stents in the innominate artery because of foreshortening on expansion of the right common carotid stent (f).

\section{Statement of Ethics}

The study protocol was approved by the hospital ethical committee and was therefore performed in accordance with the ethical standards laid down in the 1964 Declaration of Helsinki and its later amendments. Specific national laws were observed, too. Written informed consent was obtained from all patients or their legal guardians.

\section{Discussion}

SKS has been used to treat aortoiliac and/or coronary bifurcation lesions. It involves deployment of 2 appropriately sized stents, 1 in the main vessel and the other in the side branch with overlapping stents in the main vessel proximally, extending proximally to the carina of bifurcation [3]. At the bifurcation, the inflation of a single balloon in 1 artery may cause compression of the contralateral artery, resulting in relatively poor dilatation and the risk of embolization in the contralateral artery. The simultaneous inflation of bilateral balloons can avoid these problems. Additionally, the simultaneous placement of bilateral stents using the SKS technique can further decrease the incidence of these complications [4]. The SKS technique could avoid recrossing the stent struts and prevent stent crush or defor- 
Zhang et al.: Simultaneous Kissing Stenting: A Valuable Technique for Reconstructing the Stenotic Initial Segment of the Right Subclavian Artery
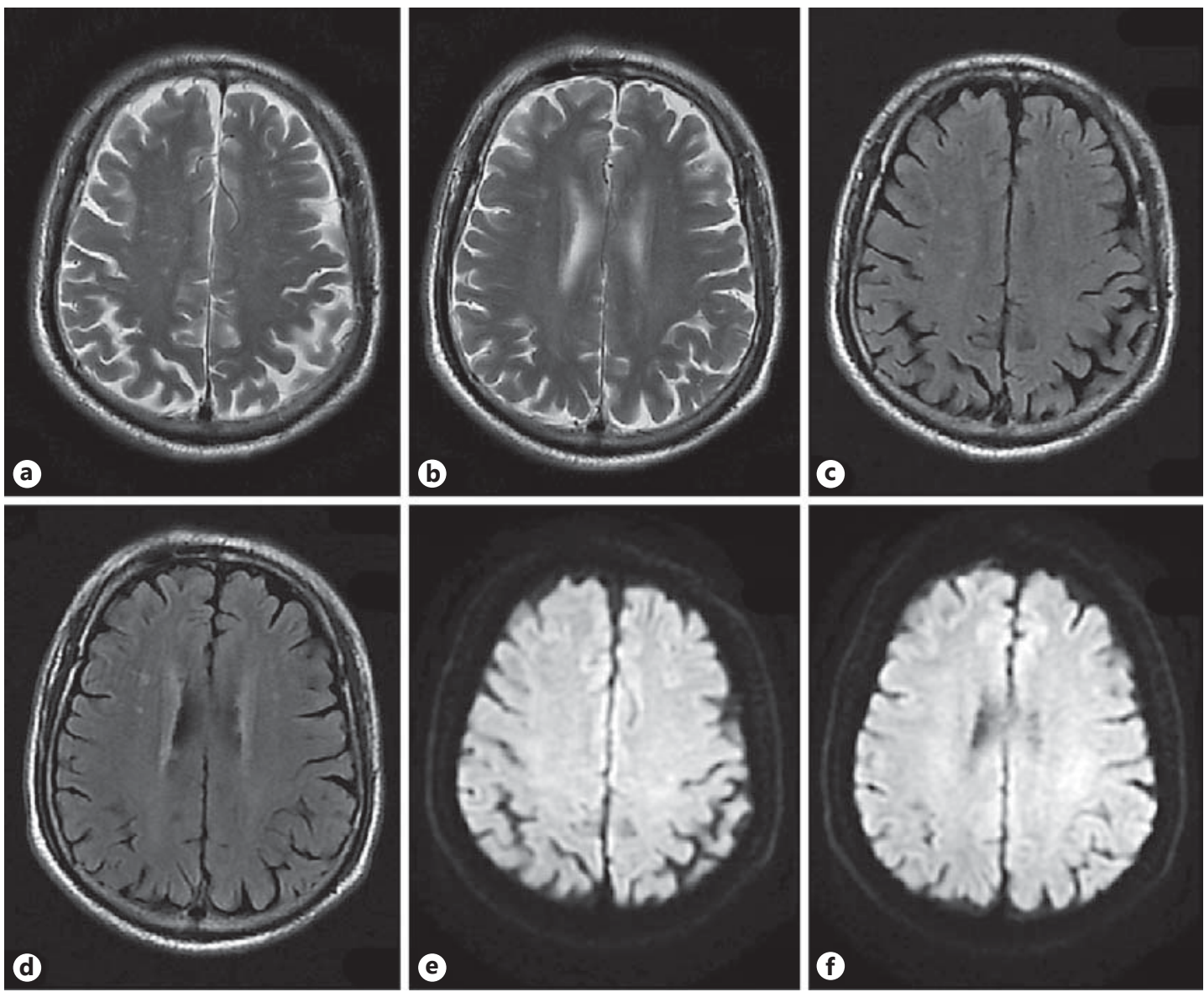

Fig. 3. MRI of Patient 2, revealing multiple lacunar infarctions of frontal lobe and parietal lobe of both sides (a, b T2; c, d T2 Flair). e, f Diffusion-weighted image showing no fresh infarctions.

mation, with guaranteed coverage of the side branch ostium [3]. Thus, in recent years, SKS has been widely applied for the percutaneous treatment of bifurcation lesions in mediumand large-size coronary arteries $[3,5]$. Till now, SKS has been rarely used in the treatment of subclavian stenosis or occlusion. Li et al. [6] reported 1 case using the SKS technique for the treatment of a subclavian artery aneurysm. In most cases of right subclavian stenosis, stenting in the single artery would be sufficient. However, when the stenosis is very close to the orifice of the right subclavian artery, in order to cover the whole lesion, the proximal end of the subclavian stent will definitely be inserted into the innominate artery. In that case, if the patient already has multiple angiostenosis or has high risks of angiostenosis and stroke involving the right carotid system, further stenting in the right carotid system will be difficult or even impossible, as the pathway from the innominate artery to the right carotid artery is blocked. Therefore, we applied the SKS technique to resolve this problem.

So far, we have successfully applied this technique in 2 patients, as described above in detail. Patient 1 suffered from multiple angiostenoses, including a mild stenosis of the right common carotid artery. Though early intervention therapy is not needed for a mild lesion, a future angioplasty or stenting, once the lesion worsens, might be necessary. Patient 2 also suffered from multiple angiostenoses, including a severe stenosis of the right internal carotid artery, which needed immediate attention. As in-stent restenosis is a complication that cannot 
Zhang et al.: Simultaneous Kissing Stenting: A Valuable Technique for Reconstructing the Stenotic Initial Segment of the Right Subclavian Artery
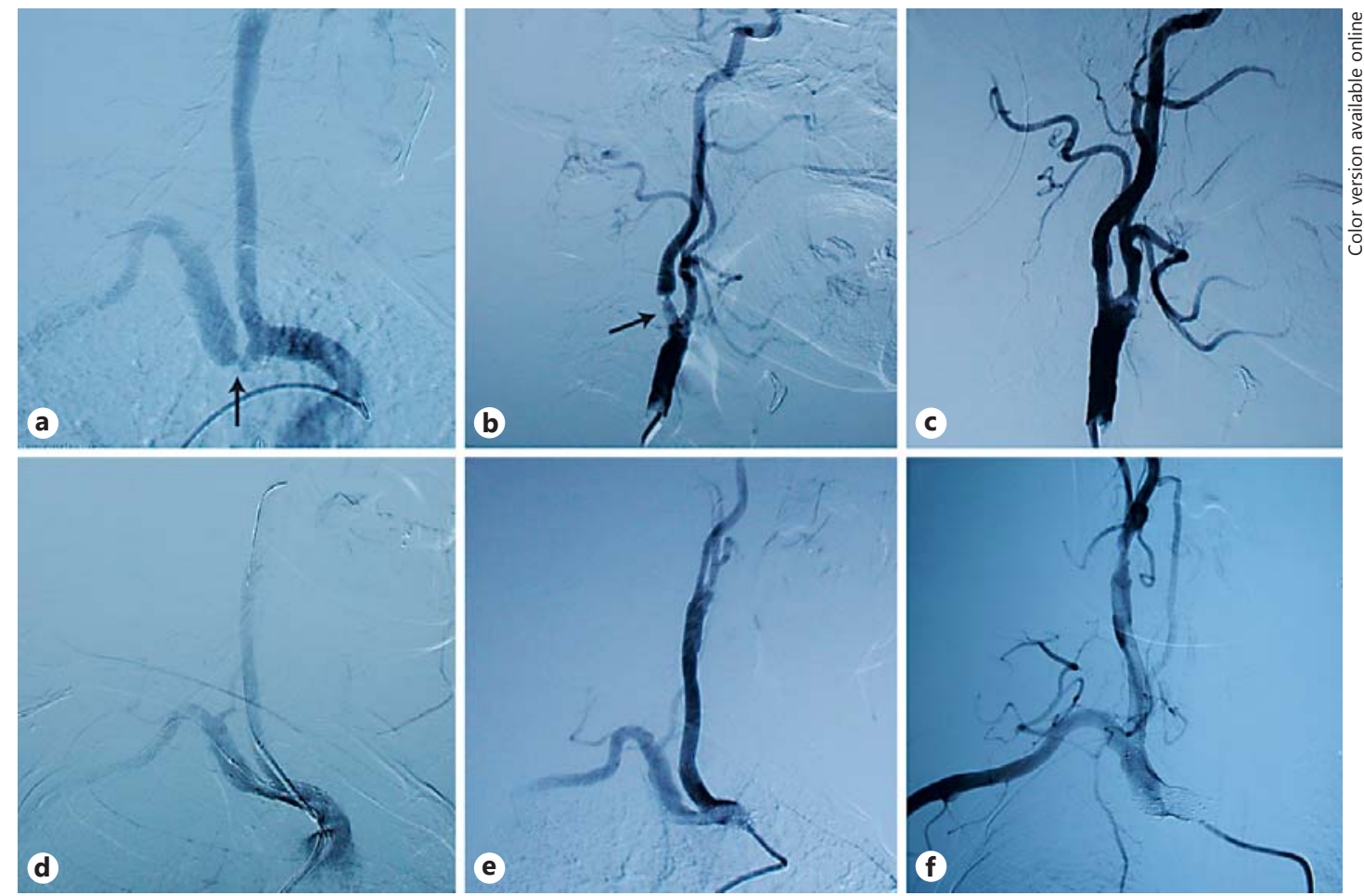

Fig. 4. Patient 2 . Angiography showing severe stenosis (about 90\%) of the initial segment of the right subclavian artery (arrow; a); severe stenosis (about 85\%) of the initial segment of the right internal carotid artery, accompanied by ulceration and thrombosis (arrow; b); a $9 \times 30 \mathrm{~mm}$ Carotid Wallstent ${ }^{\mathrm{TM}}$ being inserted to reconstruct the lumen of the right internal carotid artery under the protection of a FilterWire (c); a $9 \times 40$ mm Protégé RX being inserted into the initial segment of the right subclavian artery after the balloon angioplasty, and a $10 \times 40 \mathrm{~mm}$ Protégé RX being delivered to the initial segment of the right common carotid artery (d); excellent dilatation of the stenotic segments of the right subclavian artery and the right internal carotid artery after SKS (e), and alignment of the proximal ends of the 2 stents located in the innominate artery (f).

Fig. 5. Five-month follow-up of Patient 2. CT angiography showing excellent dilatation of the stenotic segments of the right subclavian artery and right internal carotid artery, as well as good positions of all stents $(\mathbf{a}-\mathbf{c})$.
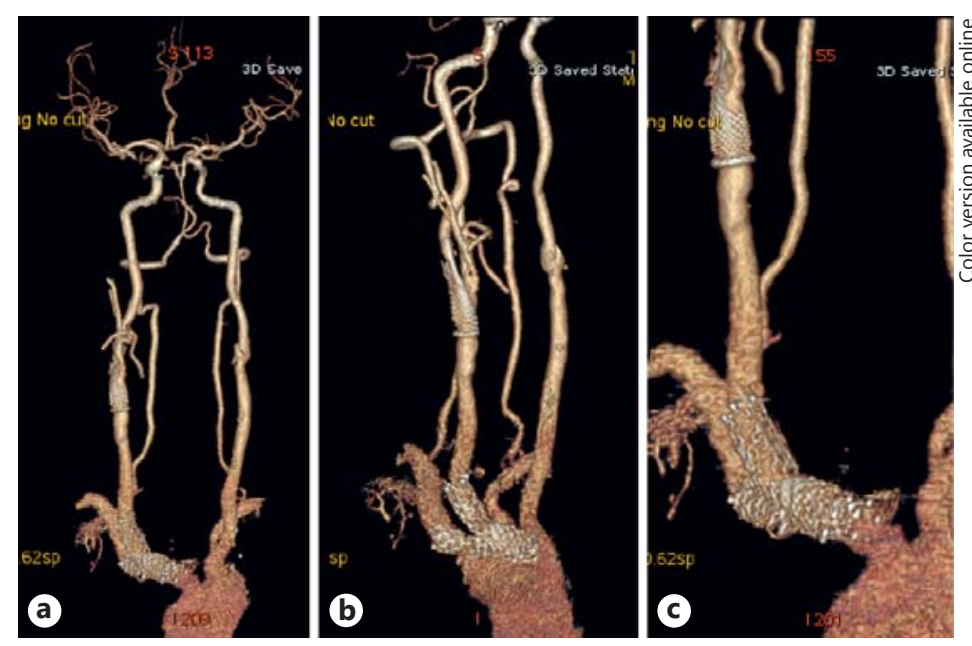
be completely avoided, the patency of the right carotid system must be maintained. In these situations, SKS could not be more suitable for a right subclavian lesion close to the orifice. In conclusion, the indication for SKS in right subclavian stenosis or occlusion is: a lesion in the initial segment of the right subclavian artery, close to the orifice, and lesions (or potential ones) in the right carotid system.

The type of stents should be chosen carefully. In our patients, because the lesions were located near the heart, self-expanding stents were selected. These were less flexible than balloon-expandable stents and not easily deformed by physical activity. In addition, the rate of restenosis using self-expanding stents is reported to be lower than the rate using balloonexpandable stents [7]. Also, self-expanding stents tend to conform to each other when apposed, as compared with balloon-expandable stents, which due to greater radial force retain their circumferential shape, leaving a potential space between the devices [8].

Depending on the density of struts, stents have a closed-cell or an open-cell configuration [9]. Closed-cell stents are characterized by small free-cell areas between struts, whereas open-cell stents have larger gaps uncovered [10]. Either open-cell or closed-cell stents may be used indiscriminately in most carotid artery stenting procedures. No significant differences in the rates of major adverse events, including stroke-death, have been reported in recent study [11]. Though closed-cell stents are less flexible and may develop kinks and incomplete expansion, they potentially offer maximal scaffolding to the vessel wall, which makes them more suitable for lesions with vulnerable plaques or suspected high embolic potential [12]. Conversely, when treating angulated vessels or tortuous anatomy, stents with a flexible and conformable open-cell configuration are preferred [12]. Additionally, less stent foreshortening on expansion is another advantage of open-cell stents because stent struts are not constrained to move along the long axis of the stent, which may allow greater precision in stent deployment [13]. In SKS technique, the proximal ends of the 2 stents should be aligned, and therefore the use of open-cell stents with limited foreshortening would be more suitable.

Carotid Wallstents (closed-cell stents) were used in Patient 1. However, the right common carotid stent became foreshortened during the self-expanding process, which prevented the alignment of the proximal ends of the 2 stents in the innominate artery. Then we tried Protégé RX stents (open-cell stents) in Patient 2 for SKS and achieved a satisfactory result. For the lesion in the right internal carotid artery of Patient 2, we chose a Carotid Wallstent, as the closed-cell stent was more suitable for the high embolic potential lesion with ulceration and thrombosis.

\section{Conclusions}

SKS of the right subclavian artery and the right carotid artery might be a safe and effective procedure when the stenotic or occlusive lesion in the initial segment of the right subclavian artery is close to the orifice, and lesions (or potential ones) exist in the right carotid system. In this way, the stenosed right subclavian artery can be dilated and the patency of the right carotid system can be maintained.

\section{Disclosure Statement}

The authors declare that they have no conflicts of interest to disclose. The authors have no personal financial or institutional interest in any of the drugs, materials, or devices described in this article. 
Zhang et al.: Simultaneous Kissing Stenting: A Valuable Technique for Reconstructing the Stenotic Initial Segment of the Right Subclavian Artery

\section{References}

1 Hass WK, Fields WS, North RR, Kircheff II, Chase NE, Bauer RB: Joint study of extracranial arterial occlusion. II. Arteriography, techniques, sites, and complications. JAMA 1968;203:961-968.

2 Chatterjee S, Nerella N, Chakravarty S, Shani J: Angioplasty alone versus angioplasty and stenting for subclavian artery stenosis - a systematic review and meta-analysis. Am J Ther 2013;20:520-523.

3 Sharma SK: Simultaneous kissing drug-eluting stent technique for percutaneous treatment of bifurcation lesions in large-size vessels. Catheter Cardiovasc Interv 2005;65:10-16.

4 Sharafuddin MJ, Hoballah JJ, Kresowik TF, Sharp WJ, Golzarian J, Sun S, Corson JD: Long-term outcome following stent reconstruction of the aortic bifurcation and the role of geometric determinants. Ann Vasc Surg 2008;22:346-357.

5 Sharma SK, Choudhury A, Lee J, Kim MC, Fisher E, Steinheimer AM, Kini AS: Simultaneous kissing stents (SKS) technique for treating bifurcation lesions in medium-to-large size coronary arteries. Am J Cardiol 2004;94: 913-917.

6 Li L, Yu H, Qi Y, Qi L, Li J, Gu Y, Zhang J: Simultaneous kissing stent technique with stent grafts for subclavian artery aneurysm: a case report. Ann Vasc Surg 2015;29:1316.e17-e19.

7 Miyakoshi A, Hatano T, Tsukahara T, Murakami M, Arai D, Yamaguchi S: Percutaneous transluminal angioplasty for atherosclerotic stenosis of the subclavian or innominate artery: angiographic and clinical outcomes in 36 patients. Neurosurg Rev 2012;35:121-126.

8 Teso D, Bloch R, Pohlman T, Karmy-Jones R: Simultaneous endovascular repair of traumatic rupture of the right subclavian artery and thoracic aorta. Ann Thorac Surg 2011;91:281-283.

9 Stoeckel D, Bonsignore C, Duda S: A survey of stent designs. Minim Invasive Ther Allied Technol 2002;11: 137-147.

10 Bosiers M, de Donato G, Deloose K, Verbist J, Peeters P, Castriota F, Cremonesi A, Setacci C: Does free cell area influence the outcome in carotid artery stenting? Eur J Vasc Endovasc Surg 2007;33:135-141.

11 Schillinger M, Gschwendtner M, Reimers B, Trenkler J, Stockx L, Mair J, Macdonald S, Karnel F, Huber K, Minar E: Does carotid stent cell design matter? Stroke 2008;39:905-909.

12 Pierce DS, Rosero EB, Modrall JG, Adams-Huet B, Valentine RJ, Clagett GP, Timaran CH: Open-cell versus closed-cell stent design differences in blood flow velocities after carotid stenting. J Vasc Surg 2009;49:602606.

13 Kreutzer J, Rome JJ: Open-cell design stents in congenital heart disease: a comparison of IntraStent vs. Palmaz stents. Catheter Cardiovasc Interv 2002;56:400-409. 